\title{
EDITORIAL Salvar la vida de las mujeres: aprovechando oportunidades perdidas ${ }^{1}$
}

\author{
Pío Iván Gómez Sánchez*
}

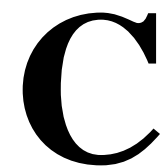

uando revisamos las cifras de morbimortalidad materna asociadas a la mala calidad de atención en los servicios clínicos o secundarias al aborto inseguro, nos vienen a la mente palabras como: impotencia, inequidad, negligencia, tragedia, injusticia social y es cuando más cobran vigencia las palabras del profesor Mahmoud Fathalla:

" Las mujeres no están muriendo por causas que no podemos evitar...están muriendo debido a que la sociedad considera que sus vidas no valen el precio de salvarlas."

La mortalidad materna es apenas la punta visible de un gran iceberg que representa una problemática mucho mas compleja. Como médicos, profesionales de la salud pública y muchos de nosotros comprometidos en el desarrollo de asistencia internacional, nos ha tomado mucho tiempo comprender la epidemiología de la enfermedad y la muerte -así como grabarnos las fallas en la prestación de los servicios de salud- dentro de un contexto mucho más amplio de derechos humanos y cambio social.

De cierta forma, estamos comprometidos en combatir una batalla -una batalla contra las actitudes injustas y desiguales acerca Del valor de las vidas humanas, particularmente, las vidas de las mujeres-. Es difícil priorizar sobre cuáles necesidades merecen recursos en medio de tantas que nos afligen. Es una lucha en la cual las mujeres son las víctimas pero también pueden ser protagonistas para hacer el cambio.

Ahora entendemos mejor el tema de la morbi-mortalidad materna en este contexto más global, y hemos comprendido que somos parte de una lucha más amplia, que necesita perspectivas nuevas para los problemas que nos aquejan hace mucho tiempo, como la mortalidad materna.

Estos viejos problemas son y seguirán siendo el centro de nuestros esfuerzos.

¿Cómo lograr que los servicios donde atendemos las complicaciones del parto o aborto sean accesibles y aceptables para las mujeres? ¿Cómo hacer que esos servicios funcionen de manera que las mujeres sean tratadas con dignidad y reciban atención médica segura y de calidad?

\section{PERSPECTIVAS MÁS AMPLIAS TRAEN CONSIGO NUEVAS AGENDAS}

Debemos ver los momentos de crisis como oportunidades de tal manera que:

Cualquier contacto con el sistema de salud sea una oportunidad para conocer más ampliamente las necesidades en atención de salud, para brindar consejería sobre elecciones, señales de alerta, o post consulta, es una buena oportunidad para educar y empoderar a las mujeres como actores de sus propia supervivencia al embarazo, y de la supervivencia de sus hijas.

A medida que las expectativas y demandas por el mejoramiento de calidad en las comunidades aumenta, los clientes se convierten en nuestros socios en el proceso de mejoramiento de la calidad de la atención. Hoy los hospitales tienen que competir con calidad en los países donde la reforma del sector salud ha implementado el concepto de servicios de calidad, así, los clientes cuentan en la comunidad su experiencia sobre calidad de la atención. De esta manera una institución puede ganar o perder reputación

\footnotetext{
* Médico Cirujano y Epidemiólogo. Universidad Del Rosario. Ginecobstetra. Universidad Nacional de Colombia. Profesor Asociado de la Universidad Nacional de Colombia e Licencia especial vinculado como Senior Medical Associate de Engenderhealth. New York

Traducción de Conferencia dictada por el Dr. Pío Iván Gómez en: Population Council conference on Post-Abortion Care Programs in Latin América. Junio 2001 en Ciudad de Guatemala.
} 
y credibilidad. Las comunidades deben involucrarse en moldear la provisión del servicio para suplir sus necesidades. En países como Colombia ya representantes de la comunidad hacen parte de las juntas directivas de los hospitales.

La calidad va más allá de servicios médicos seguros; también incluye respeto por la dignidad humana y los derechos humanos. Calidad implica brindar servicios integrales, que satisfagan una serie de necesidades de la mujer, son una oportunidad para hacer mucho más y no deben limitarse al componente orgánico. Como profesionales de la salud hemos olvidado que el ser humano tiene no solo cuerpo sino mente y además factores sociales, familiares, ambientales, económicos etc., que pueden afectar la salud. No debemos olvidarnos del modelo bio-psico-social en la conformación de servicios integrales. Debemos dejar de enseñar en nuestras escuelas de medicina modelos curativos y enfatizar en la importancia de la promoción de la salud y la prevención de la enfermedad.

Como anécdota, recuerdo una paciente que asistió a una clínica oncológica cuando en el Instituto Materno Infantil de Bogotá hacía mi segundo año de Residencia en Ginecología. Ella era una mujer joven con 6 hijos y con un cancer infiltrante de cuello uterino, mi primera reacción fue de rabia pues para mi era difícil entender como nunca se había realizado una citología. Al preguntarle, su respuesta aún está en mi cabeza: "Doctor, yo he tenido todos mis partos en este Hospital y nunca nadie me dijo que era eso de la citología".

Que doloroso que a diario perdamos oportunidades de salvar la vida de las mujeres; si alguien hubiera hablado con ella de la citología quizás no hubiera muerto. Cuántas veces no tratamos abortos incompletos y nos limitamos a realizar la evacuación uterina y la mujer se va sin saber que en los próximos once días ovulará de nuevo; se va sin conocer los métodos de planificación familiar; sin saber a donde ir para conseguirlos; sin saber como puede evitar una Infección de Transmisión Sexual; sin conocer que existe la citología cervicovaginal, el autoexamen de seno, etc. Todas estas son oportunidades perdidas.

No sé en que momento dejamos de ser médicos para convertirnos en simplemente técnicos que saben reparar (y a veces mal) un organismo. ¿Dónde queda el componente humano? A veces en países como el mío, culpamos los nuevos Sistemas de Seguridad Social en Salud, pero ¿son estos los culpables? Creo que no; la culpa no es de los Sistemas sino de la actitud de los proveedores de los servicios, quienes en cualquier sistema, si así lo desean pueden brindar servicios cálidos y con calidad.

Estoy hablando de fortalecer la calidad de la atención y hacer los servicios más humanos mientras se lleva a cabo cualquier consulta por siempre que sea, cualquier tratamiento, en fin, en cualquier contacto en los servicios de salud.

\section{SALVAR LA VIDA DE LA MUJER ES NECESARIO PERO NO SUFICIENTE}

Cada año, cerca de 600.000 mujeres mueren por complicaciones relacionadas con la gestación. La gran mayoría de estas muertes podrían ser relativamente fáciles de evitar si tuviéramos la voluntad de aprovechar las oportunidades perdidas aún con recursos, mínimos en nuestras instituciones.

Para hacerlo debemos preocuparnos más por las mujeres y demostrar nuestro interés por entender sus necesidades y exigir que reciban atención de alta calidad. Se conoce que 46 millones de mujeres en todo el mundo presentan abortos cada año. De estas mujeres, $78 \%$ viven en países en vía de desarrollo y $22 \%$ en países desarrollados. La Organización Mundial de la Salud y otras instituciones estiman que en América Latina existen las tasas más altas de aborto inseguro en el mundo: 4.6 millones de abortos inseguros tienen lugar anualmente, es decir, que 40 de cada 1.000 mujeres en edad reproductiva, tienen abortos inseguros. El aborto inseguro causa un cuarto de las muertes maternas en la región, 6.000 cada año. Estas estadísticas señalan la muerte relacionada con el aborto, una tragedia que sólo puede evitarse cambiando actitudes, estructura de los servicios y políticas.

Al suministrar servicios básicos costo-efectivos, podemos salvar las vidas de las mujeres, pero para poder lograr los objetivos que nosotros mismos nos hemos fijado en Cairo y Beijín, debemos hacer mucho más para ayudar a las mujeres a conocer sus derechos sexuales y reproductivos. 
Hoy no definimos salud como la ausencia de enfermedad, así tampoco basta con sólo salvar una vida, sino también hay que mejorar la condición de vida y bienestar de las mujeres.

Cada vez que vemos a una mujer por un caso relacionado con embarazo, tenemos la oportunidad de marcar una diferencia en su vida.

\section{LA CLIENTE NO ES UN ÚTERO}

Esto comienza por ir más allá de los aspectos clínicos de traer al mundo un bebé, o manejar una complicación de parto o aborto. A menudo, los proveedores se centran en el procedimiento clínico sin tomar en consideración un punto de vista más amplio de la cliente. Es triste ver en nuestros hospitales como se habla de la mujer "de la cama 35", "la de la histerectomía", "la del aborto provocado"...

A menudo las mujeres vienen a nuestras instalaciones y se van sin haber tenido la oportunidad de explicar sus necesidades y temores, mucho menos de haber expresado sus sueños para el futuro.

Recuerdo alguna vez ayudando a un residente de tercer año de Ginecobstetricia qien estaba desconcertado ante el caso de una mujer que habia consultado reiteradamente por flujo vaginal y nunca le encontraban flujo al examen. Reinterrogamos la paciente en un ambiente de privacidad y de confianza logrando que la mujer expusiera su verdadero motivo de consulta: "no siento placer al estar con mi esposo", era un caso de disfunción sexual que atormentaba a la mujer pero ella tenía miedo de hablar de esto por temor a ser juzgada y esperaba que al decir que tenía flujo vaginal, el médico la examinaría y encontraría así la causa de su disfunción sexual. Esto es apenas un pequeño ejemplo de lo difícil que es ayudar las mujeres si sólo nos centramos en lo orgánico; por eso siempre les dije a mis estudiantes: "ustedes se están formando para ser Ginecólogos, no Genitólogos, es decir expertos en la mujer no sólo en sus genitales. Sólo si entendemos que el ser humano es un ser Bio-Psico-Social y de esta forma enfocamos nuestros servicios médicos, estaremos brindando atención de buena calidad.

\section{LO QUE NO SE VE PUEDE MATAR}

Las prácticas inadecuadas de prevención de infecciones son causantes de tasas inaceptablemente altas de morbilidad y mortalidad de las mujeres. La iniciativa de Maternidad Segura sugiere que actualmente, el $15 \%$ de la mortalidad materna global es atribuible a infecciones nosocomiales.

Muchos de los triunfos de la salud pública del siglo 20 son directamente atribuibles a las prácticas efectivas de prevención de infecciones. Medidas tan simples como un lavado de manos, uso de soluciones de Cloro, y manejo adecuado de los desperdicios han resultado en reducciones significativas de mortalidad materna, enfermedades infecciosas en los niños, y enfermedades adquiridas en hospitales. Por ejemplo en USA, durante el siglo 20, se produjo una reducción Del 98\% en la mortalidad materna y un $70 \%$ en las muertes maternas atribuibles a infecciones como resultado del mejoramiento en las prácticas de prevención de infecciones. Si somos conscientes de la importancia de prácticas adecuadas de prevención de infecciones para proteger a nuestros usuarios(as) y a nosotros mismos, entonces por que en nuestras instituciones no lo hacemos, si además son medidas poco costosas?

Reflexionemos sobre nuestro quehacer diario:

¿Nos lavamos las manos antes y después de atender un(a) cliente(a)?

¿Nos protegemos nosotros mismos? ¿Usamos soluciones de cloro para descontaminar los elementos que han tenido contacto con secreciones o sangre? ¿Usamos anteojos protectores?

¡Que diferncia hay entre nuestro discurso teórico y la práctica!

\section{PROVEEDORES Y CLIENTES TIENEN PERCEPCIONES DIFERENTES DEL DOLOR}

A menudo los médicos subestimamos el dolor físico y emocional asociado con el parto o la pérdida del embarazo. Aún es común ver en las órdenes postoperatorias la nota: "Analgésicos según dolor", esto 
es como si prescribieramos antibióticos según infección. En muchos sitios algunos proveedores no son amigos de colocar analgesia peridural en el trabajo de parto. Si estos dos ejemplos se dan en postoperatorios donde se reconoce que el dolor es importante, ¿qué podremos decir del tratamiento del aborto?

Miremos algunas anécdotas:

- Un grupo de proveedores de la salud en Pakistán explicaban que no se utilizaba anestesia para el control Del dolor durante procedimientos de curetaje, debido a que "el proceso no es tan doloroso".

- Cuando se le preguntó a una mujer de Uganda, cuales eran sus inquietudes antes del curetaje, su principal temor era el dolor que sentiría durante el procedimiento. Cuando se le dijo que podría sentir algo de incomodidad, contestó: "Yo los conozco a ustedes, doctores, cuando dicen que habrá un ligero dolor, significa que dolerá mucho".

Los requisitos generales para control del dolor formulan: "Todas las sensaciones de dolor se aumentan con el miedo; la atención constante y la confianza por medio del tacto y palabras ("anestesia verbal o verbocaína") son importantes para ayudar a la mujer a enfrentar esta parte de la experiencia."

Esto significa que una mujer que está ansiosa o que es ignorada durante el tratamiento, padecerá más dolor que una que cuenta con el apoyo y la atención del proveedor del servicio. Así también, una clara explicación de las sensaciones y molestias que una cliente podrá llegar a sentir, puede mejorar el manejo del dolor. Entender el dolor de una mujer y ayudarle a vencer sus temores puede hacer una diferencia dramática en el nivel de dolor que experimenta psicológica y físicamente.

Sabemos que en algunos casos, los proveedores manejan mal el dolor sobredosificando a las mujeres para no tenerlas despiertas -una mujer que está despierta durante el tratamiento de una complicación de aborto requiere apoyo adicional durante el procedimiento para ayudarle a manejar el dolor-.

Por otro lado, algunos proveedores prestan atención insuficiente al control del dolor bien sea porque subestiman el dolor que una mujer puede sentir durante una evacuación, o bien porque creen que mientras más sufre una mujer durante el procedimiento, menos probabilidad habrá de que repita una complicación por aborto. No hay evidencia de que el dolor durante el parto o el aborto reduzca su incidencia en un embarazo posterior deseado o no deseado. Lo que si se ha comprobado que ayuda a las mujeres, es la atención compasiva la consejería apropiada en planificación familiar después del embarazo.

\section{SIEMPRE HAY TIEMPO PARA SER AMABLE}

Cuando una enfermera en Colombia supo cómo podía mitigar el dolor de alguien con tan solo tocarla, comenzó a tomar las manos de la mujer y a darle palabras de aliento. Antes de esto, la misma enfermera permanecía a un lado durante los procedimientos con anestesia local, observando periódicamente el monitor paa vigilar los signos vitales de la paciente. Una vez que la enfermera conoció un modelo de consejería de apoyo durante procedimientos que se realizan con anestesia local, aprendió que escuchar todas las inquietudes de las pacientes, incluyendo la necesidad de controlar el dolor y recibir apoyo emocional, era una ayuda que ella podía ofrecer dentro del alcance de su trabajo.

La consejería no precisa de tiempo especial para sostener una larga discusión en un salón privado. Consejería significa responder a las necesidades de las mujeres por información y apoyo emocional, bien sea que dichas necesidades surjan antes, durante o después del procedimiento. Tomarse un momento para llamar a la cliente por su nombre, preguntarle cuáles son sus inquietudes y darle información acerca de cómo cuidarse (a corto y largo plazo) puede tener un impacto profundo en la experiencia de hospitalización de una mujer y en su vida futura. Esos pocos minutos dedicados a conocer las necesidades de la mujer y ayudarle a solucionarlas, puede hacer que ésta transforme el concepto de su propio cuerpo, sus derechos y su calidad de vida. Tomarse tiempo para ser "amable" puede aumentar la retribución de la inversión del proveedor en la atención dada a las mujeres. 


\section{ENTONCES, ¿POR QUE NO PRESTAR A LA MUJER EMBARAZA, SERVICIOS DE MEJOR CALIDAD, MÁS HUMANOS Y MÁS COMPLETOS?}

Nosotros, los profesionales de la salud, estamos dedicados a mejorar la calidad de la vida de las mujeres a las que servimos, pero a menudo nuestros ideales no se realizan. Con frecuencia nos preocupa pensar que no tenemos suficiente tiempo para prestar una mejor atención, pero la crisis puede convertirse en oportunidad.

Hoy día, en la gerencia moderna, los momentos de crisis se ven no como problemas sino como oportunidades. El embarazo puede impulsar a la mujer a confrontar sus propios deseos e inclinaciones acerca de su vida reproductiva. Este es un punto crítico sobre el cual las mujeres pueden hacer elecciones sobre cómo controlar su propia fertilidad, tanto a corto como a largo plazo. El proveedor que se encuentra con una mujer en este período de tiempo tiene una enorme oportunidad de afectar la vida de ésta y su futuro. La atención prestada para informar, apoyar y empoderar a esa mujer, puede tener un efecto duradero y multiplicador -uno que puede permitirle tomar el control no solo de su salud reproductiva, sino también de sus necesidades personales, físicas, emocionales, educativas y espirituales. La crisis la puede acercar al proveedor, pero el proveedor puede hacer mucho más que solamente sacarla de la crisis-ese proveedor tiene la oportunidad de cambiar su vida para mejorarla.

Ada (seudónimo), mujer colombiana de 29 años, es soltera, tiene una hija de una año y educación hasta octavo grado. Vive con su madre y un hermano. Cuando supo que estaba embarazada decidió terminar con el embarazo "porque soy una madre soltera y el hombre que me embarazó no quería tener el bebé". Debido a que el aborto es ilegal, consultó con sus amigas, quienes le dijeron que podía tomar medicinas para así, darlo por terminado. Ella dijo:"sabía lo que estaba haciendo, muy en el fondo yo no quería perderlo (el embarazo) pero me estaba volviendo loca, me sentía desesperada". Cuando ingirió las pildoras dijo que tuvo fuertes dolores, "como si me fuera a llegar el período". Tenía dolor adelante y atrás. Pensé que iba morir... "Emocionalmente estaba mal ya que no estaba de acuerdo con el aborto, pero mi situación me hizo hacerlo.." Sangró más de lo que esperaba y se asustó tanto que decidió acudir sola al hospital.

En el hospital la entrevistamos después de ser tratada por un aborto incompleto. Acerca de su experiencia, comenta: "no fue tan buena". Parte del personal fue amable y parte no. Tuve que rogar para que me atendieran y tuve que esperar mucho tiempo. No quiero volver, no me entendían, nadie me quería atender. Sufro porque esto duele, estoy cansada de esperar, triste, culpable por haberlo hecho, pero no tenía otra elección, ahora, solo espero salir pronto, volver a casa".

¿Será que con la atención recibida, esta mujer evitará un nuevo embarazo? ¿Le dimos las herramientas para evitarlo?; ¿para protegerse de ITS?; ¿aprovechamos la oportunidad para darle consejería en aspectos importantes de Salud Sexual y Reproductiva? La respuesta es No. Se perdió una muy buena oportunidad.

\section{A LO ÚNICO A QUE HAY QUE TEMER, ES AL MIEDO MISMO}

Aún los proveedores con las mejores intenciones pueden no comprender lo que una mujer vive antes de llegar al hospital.

Sarah, una enfermera partera de Uganda relata la historia de una cliente en su hospital, con complicaciones por aborto séptico. La mujer esperó durante cuatro días antes de acudir a un tratamiento médico, y cuando llegó al hospital, su condición ya era muy avanzada para que los proveedores la pudieran salvar. "¿porqué no vino antes?", se preguntó Sarah con frustración. "la habríamos podido ayudar si no hubiera esperado tanto". A través de la capacitación, Sarah aprendió cómo las habilidades de comunicación de los proveedores tienen un impacto dramático en la dignidad, confidencialidad, y privacidad de las clientes postaborto. Cuando vio cómo las conductas más simples de los proveedores afectan la experiencia de hospitalización de una clienta de postaborto, Sarah fue consciente de la importancia de ofrecer a todos los clientes el máximo apoyo y respeto, y manifestó: "pienso que ahora entiendo porqué las clientas con complicaciones de aborto esperan tanto para venir al hospital: tienen miedo de cómo las trataremos". 
El miedo es la razón principal de porqué las mujeres se demoran en buscar tratamiento; temen que otros conocerán su condición, que serán reportadas a la polícia por haber inducido ilegalmente el aborto, que serán maltratadas por los proveedores. Al mismo tiempo, el miedo a morir es lo que a menudo lleva a las mujeres a buscar atención en un hospital. Pero, como en la historia que Sarah compartió, también pueden esperar mucho. Cuando los proveedores irrespetan a la mujer que llega al servicio, el comentario se riega rápidamente entre la comunidad. Así, las mujeres demorarán el tratamiento y podrán sufrir o morir como resultado de ese temor. El miedo se basó en la realidad, pero innecesaria. Los proveedores pueden eliminar las razones para muchos de esos temores.

Es hora de reflexionar sobre nuestra actitud como proveedores de servicios de salud: ¿seguiremos enfocados en esquemas terapéuticos orgánicos? o ¿trataremos de enfocarnos en la prevención?

Seguiremos obsesionados, como pasa en mi país, en la facturación de servicios (producir o desaparecer) o ¿volveremos a la senda del médico "antiguo": humano, cálido, amigo y confidente.

Creo firmemente que los cambios en Seguridad Social en Salud que se han dado y se seguirán dando en el mundo no riñen con la atención de calidad y humanizada, ;todo es cuestión de actitud y de querer cambiar! 